\title{
Outils d'évaluation pour apprendre et enseigner avec succès
}

Les hôpitaux sont des organisations apprenantes qui doivent s'adapter aux besoins de la société dans un environnement caractérisé par des ressources limitées en personnel et par l'augmentation des malades chroniques et des coûts du progrès de la technique médicale. Prodiguée essentiellement en milieu hospitalier, la formation postgraduée médicale subit, dans ce «triangle des Bermudes», une forte pression amplifiée par l'introduction du système DRG. Or aucun hôpital ne pourrait fonctionner sans médecin. Il est donc absolument nécessaire d'investir dans la formation postgraduée médicale pour garantir à la population des soins de haute qualité et assurer la survie des hôpitaux.

La Commission pour la formation postgraduée et continue (CFPC) introduit actuellement un système d'évaluation centrée sur le lieu de travail en vue d'optimiser la qualité de la formation postgraduée médicale et de lui donner une structure plus efficace. Ces évaluations permettent aux apprenants de contrôler leur niveau de connaissances et de détecter leurs lacunes éventuelles. Elles sont formatives pendant toute la formation postgraduée et sommatives au terme de celle-ci. Une collaboration a démarré avec l'Institut de formation médicale de l'Université de Berne pour mettre au point différents instruments d'évaluation applicables pendant toute la formation professionnelle médicale (des études universitaires à la formation continue en passant par la formation postgraduée). Ces instruments sont, par exemple, les «Mini Clinical Evaluation Exercises» et la «Direct Observation of Procedural Skills» ainsi que la plate-forme Internet pour les questions à choix multiple et les questions à réponses brèves pour les examens de spécialiste.

Dans la présente édition du BMS, nous vous présentons les premiers résultats de l'évaluation de la formation postgraduée centrée sur le lieu de travail [1]. L'introduction de ces instruments d'évaluation dans les établissements de formation postgraduée (hôpitaux) a déjà, à elle seule, induit un apprentissage. Les responsables de la formation (cadres médicaux) sont conduits à mettre en question les processus centraux de l'organisation interne et à les restructurer, le cas échéant. Ces processus sont, entre autres, les suivants: anamnèses, examens cliniques, information au patient sur les interventions diagnostiques et thérapeutiques, constats, médication, déroulement des opérations et gestion des risques et des fautes. Les services et départements impliqués définissent des déroulements standards, intensifient l'enseignement clinique et supervisent les médecins-assistants dans la prise en charge des patients. Ce qui induit évidemment une amélioration de la qualité des soins. Les instruments d'évaluation et le feedback relatifs à la formation dispensée conduisent à une structuration accrue, laquelle augmente à son tour la qualité et l'efficacité de la formation postgraduée médicale. Les médecins-assistants reçoivent un feedback sur l'état de leurs compétences cliniques, ce qui leur permet d'apprendre de manière ciblée; la communication au sein de l'organisation s'en trouve directement améliorée.

Les médecins-assistants comme les hôpitaux tirent profit de l'évaluation centrée sur le lieu de travail et du feedback. Ce processus, dans le cadre duquel certains médecins-assistants et médecins-cadres sont conduits à vivre des situations difficiles [2], est constitutif de l'apprentissage organisationnel. Forte des premières expériences positives faites avec ce système d'évaluation, la CFPC a décidé de l'introduire, si possible, dans tous les établissements de formation postgraduée. A l'occasion de l'assemblée plénière du 20 novembre 2008, des experts renommés des Etats-Unis, des Pays-Bas et de Grande-Bretagne parleront de leurs propres expériences. La CFPC en tiendra compte dans la mise en œuvre, de manière à convaincre tous les enseignants de Suisse concernés par la formation postgraduée médicale et à gagner leur collaboration.

$$
\begin{array}{r}
\text { Dr Max Giger, } \\
\text { membre du Comité central de la FMH } \\
\text { et président de la Commission } \\
\text { pour la formation postgraduée et continue }
\end{array}
$$

1 Berendonk C, Beyer C, Westkämper R, Giger M. Le feedback structuré dans la formation postgraduée médicale: Mini-CEX et DOPS. Bull Méd Suisses. 2008;89(32):1337-40.

2 Argyris C, Schön DA. Die lernende Organisation. Stuttgart: Klett-Cotta; 1999. 